\title{
The diffusion path reversibility confirms symmetry of surface barriers
}

\author{
German Sastre $^{1}$, Jörg Kärger ${ }^{2}$, Douglas M. Ruthven ${ }^{3 *}$ \\ ${ }^{1}$ Instituto de Tecnologia Quimica U.P.V.-C.S.I.C., Universidad Politecnica de Valencia, Spain \\ ${ }^{2}$ Faculty of Physics and Earth Sciences, University of Leipzig, Leipzig, Germany \\ ${ }^{3}$ Department of Chemical and Biological Engineering, University of Maine, Orono, Maine, \\ United States \\ *DRuthven@umche.maine.edu
}

The resistance perceived by guest molecules upon entering or leaving a microporous host material (the "surface barrier") is known to often surpass the influence of diffusion in its interior on the overall rate of molecular uptake and release [1,2]. Our knowledge of the relevant mechanisms of surface permeation, however, is still rather limited. This is related to the difficulties in its direct measurement which, given the "nanoscopic" extension of the relevant space scale, notably exceed the difficulties which do exist with already the diffusion measurement. A question discussed in this context refers to the possibility that the resistance perceived by the molecules on entering the pore space may differ from that perceived upon leaving [3-5].

In first MD simulations in zeolite MFI with partial surface blockade using benzene as a probe molecule [6], with increasing surface blockage molecular exchange rates were found to be slowed down following the relevant relations adopted from effective medium theory [7]. The diffusion path lengths covered during the simulations, however, turned out to be too short for yielding a sufficiently large enough number of adsorption-desorption events.

The situation totally changed on considering methane as a probe molecule. Now many thousands of adand desorption paths could be followed. They confirmed complete symmetry of the diffusion paths during adsorption and desorption and, hence, of also the surface resistance [8].

\section{References}

[1] J. Kärger, D. M. Ruthven: Diffusion in nanoporous materials: Fundamental principles, insights and challenges. New J. Chem. 40(5), 4027-4048 (2016).

[2] J. C. S. Remi, A. Lauerer, C. Chmelik, I. Vandendael, H. Terryn, G. V. Baron, J. Denayer, J. Kärger: The role of crystal diversity in understanding mass transfer in nanoporous materials. Nat. Mater. 15(4), 401-406 (2015).

[3] A. R. Teixeira, X. Qi, C.-C. Chang, W. Fan, C. W. Conner, P. J. Dauenhauer: On asymmetric surface barriers in MFI zeolites revealed by frequency response. J. Phys. Chem. C 118, 22166-22180 (2014).

[4] D. M. Ruthven, J. Kärger: Comment on "On asymmetric surface barriers in MFI zeolites revealed by frequency response”. J. Phys. Chem. C 119(52), 29201-29202 (2015).

[5] P. J. Dauenhauer: Reply to Comment on "On asymmetric surface barriers in MFI zeolites revealed by frequency response". J. Phys. Chem. C 119(52), 29203-29205 (2015).

[6] G. Sastre, J. Kärger, D. M. Ruthven: Molecular dynamics study of diffusion and surface permeation of benzene in silicalite. J. Phys. Chem. C 122(13), 7217-7225 (2018).

[7] F. Hibbe, J. Caro, C. Chmelik, A. Huang, T. Kirchner, D. M. Ruthven, R. Valiullin, J. Kärger: Monitoring molecular mass transfer in cation-free nanoporous host-crystals of type AlPO-LTA. J. Am. Chem. Soc. 134, 7725-7732 (2012).

[8] G. Sastre, J. Kärger, D. M. Ruthven: in preparation 